\title{
Iterative methods for mixed equilibrium problems and strictly pseudocontractive mappings
}

Jong Soo Jung*

\section{"Correspondence: jungjs@dau.ac.kr; jungjs@mail.donga.ac.kr Department of Mathematics, Dong-A University, Busan, 604-714, Korea}

\begin{abstract}
In this paper, we introduce new implicit and explicit iterative schemes for finding a common element of the set of solutions of the mixed equilibrium problem and the set of fixed points of a $k$-strictly pseudocontractive non-self mapping in Hilbert spaces. We establish results of the strong convergence of the sequences generated by the proposed schemes to a common point of two sets, which is a solution of a certain variational inequality. Our results extend and improve the corresponding results given by many authors recently in this area.
\end{abstract}

MSC: 47H05; 47H06; 47H09; 47H10; 47J25; 47J05; 49M05

Keywords: mixed equilibrium problem; $k$-strictly pseudocontractive mapping; nonexpansive mapping; fixed points; $\rho$-Lipschitzian and $\eta$-strongly monotone operator; variational inequality

\section{Introduction}

Let $H$ be a real Hilbert space with inner product $\langle\cdot, \cdot\rangle$ and induced norm $\|\cdot\|$. Let $C$ be a nonempty closed convex subset of $H$ and $S: C \rightarrow C$ be a self-mapping on $C$. We denote by $F(S)$ the set of fixed points of $S$, that is, $F(S):=\{x \in C: S x=x\}$.

Let $\Theta$ be a bifunction of $C \times C$ into $\mathbb{R}$ and $\varphi: C \rightarrow \mathbb{R}$ be a function, where $\mathbb{R}$ is the set of real numbers. Then we consider the following mixed equilibrium problem (for short, MEP): finding $x \in C$ such that

$$
\Theta(x, y)+\varphi(y)-\varphi(x) \geq 0, \quad \forall y \in C,
$$

which was studied by Ceng and Yao [1] (see also [2]). The set of solutions of the MEP (1.1) is denoted by $\operatorname{MEP}(\Theta, \varphi)$. We see that $x$ being a solution of the problem (1.1) implies that $x \in \operatorname{dom} \varphi=\{\varphi(x)<\infty\}$.

If $\varphi=0$, then the MEP (1.1) becomes the following equilibrium problem (for short, EP): finding $x \in C$ such that

$$
\Theta(x, y) \geq 0, \quad \forall y \in C .
$$

The set of solutions of the EP (1.2) is denoted by $\operatorname{EP}(\Theta)$.

( 2012 Jung: licensee Springer. This is an Open Access article distributed under the terms of the Creative Commons Attribution License (http://creativecommons.org/licenses/by/2.0), which permits unrestricted use, distribution, and reproduction in any medium, provided the original work is properly cited. 
The MEP (1.1) is very general in the sense that it includes, as special cases, fixed point problems, optimization problems, variational inequality problems, minmax problems, Nash equilibrium problems in noncooperative games and others; see, e.g., [1, 3-5].

The class of pseudocontractive mappings is one of the most important classes of mappings among nonlinear mappings. Recently, many authors have devoted their studies to the problems of finding fixed points for pseudocontractive mappings; see, for example, [69] and the references therein. We recall that a mapping $S: C \rightarrow H$ is said to be $k$-strictly pseudocontractive if there exists a constant $k \in[0,1)$ such that

$$
\|S x-S y\|^{2} \leq\|x-y\|^{2}+k\|(I-S) x-(I-S) y\|^{2}, \quad \forall x, y \in C .
$$

Note that the class of $k$-strictly pseudocontractive mappings includes the class of nonexpansive mappings as a subclass. That is, $S$ is nonexpansive (i.e., $\|S x-S y\| \leq\|x-y\|$, $\forall x, y \in C)$ if and only if $S$ is 0 -strictly pseudocontractive. The mapping $S$ is also said to be pseudocontractive if $k=1$, and $S$ is said to be strongly pseudocontractive if there exists a constant $\lambda \in(0,1)$ such that $S-\lambda I$ is pseudocontractive. Clearly, the class of $k$-strictly pseudocontractive mappings falls into the one between classes of nonexpansive mappings and pseudocontractive mappings. Also, we remark that the class of strongly pseudocontractive mappings is independent of the class of $k$-strictly pseudocontractive mappings (see $[10,11])$.

Recently, in order to study the EP (1.2) coupled with the fixed point problem, many authors have introduced some iterative schemes for finding a common element of the set of solutions of the EP (1.2) and the set of fixed points of a countable family of nonexpansive mappings or strictly pseudocontractive mappings; see [12-14] and the references therein.

On the other hand, in 2001 Yamada [15] introduced the hybrid iterative method for the nonexpansive mapping to solve a variational inequality related to a Lipschitzian and strongly monotone operator. Since then, by using the ideas of Marino and Xu [16], Tien [17, 18] and Ceng et al. [19] provided the general iterative schemes for finding a fixed point of the nonexpansive mapping, which is a solution of a certain variational inequality related to a Lipschitzian and strongly monotone operator. Cho et al. [7] and Jung $[8,20]$ gave the general iterative schemes for finding a fixed point of the $k$-strictly pseudocontractive mapping, which is a solution of a certain variational inequality.

Inspired and motivated by the above mentioned recent works, in this paper, we introduce new implicit and explicit iterative schemes for finding a common element of the set of the solutions of the MEP (1.1) and the set of fixed points of a $k$-strictly pseudocontractive mapping. Then we establish results of the strong convergence of the sequences generated by the proposed schemes to a common point of two sets, which is a solution of a certain variational inequality. Our results extend and improve the recent well-known results in this area.

\section{Preliminaries and lemmas}

Let $H$ be a real Hilbert space, and let $C$ be a nonempty closed convex subset of $H$. In the following, we write $x_{n} \rightarrow x$ to indicate that the sequence $\left\{x_{n}\right\}$ converges weakly to $x$. $x_{n} \rightarrow x$ implies that $\left\{x_{n}\right\}$ converges strongly to $x$.

Recall that the mapping $V: H \rightarrow H$ is said to be $l$-Lipschitzian if

$$
\|V x-V y\| \leq l\|x-y\|, \quad \forall x, y \in H,
$$


and that the nonlinear operator $F: H \rightarrow H$ is said to be $\rho$-Lipschitzian and $\eta$-strongly monotone, where $\rho>0$ and $\eta>0$ are constants, if

$$
\|F x-F y\| \leq \rho\|x-y\|
$$

and

$$
\langle F x-F y, x-y\rangle \geq \eta\|x-y\|^{2}, \quad \forall x, y \in H .
$$

In a real Hilbert space $H$, we have

$$
\|x-y\|^{2}=\|x\|^{2}+\|y\|^{2}-2\langle x, y\rangle,
$$

for all $x, y \in H$ and $\lambda \in \mathbb{R}$. For every point $x \in H$, there exists a unique nearest point in $C$, denoted by $P_{C} x$, such that

$$
\left\|x-P_{C} x\right\| \leq\|x-y\|
$$

for all $y \in C . P_{C}$ is called the metric projection of $H$ onto $C$. It is well known that $P_{C}$ is nonexpansive and $P_{C}$ is characterized by the property

$$
u=P_{C} x \quad \Leftrightarrow \quad\langle x-u, u-y\rangle \geq 0, \quad \forall x \in H, y \in C .
$$

It is also well known that $H$ satisfies the Opial condition; that is, for any sequence $\left\{x_{n}\right\}$ with $x_{n} \rightarrow x$, the inequality

$$
\liminf _{n \rightarrow \infty}\left\|x_{n}-x\right\|<\liminf _{n \rightarrow \infty}\left\|x_{n}-y\right\|
$$

holds for every $y \in H$ with $y \neq x$.

For solving the equilibrium problem for a bifunction $\Theta: C \times C \rightarrow \mathbb{R}$, let us assume that $\Theta$ and $\varphi$ satisfy the following conditions:

(A1) $\Theta(x, x)=0$ for all $x \in C$;

(A2) $\Theta$ is monotone, that is, $\Theta(x, y)+\Theta(y, x) \leq 0$ for all $x, y \in C$;

(A3) for each $x, y, z \in C$,

$$
\lim _{t \downarrow 0} \Theta(t z+(1-t) x, y) \leq \Theta(x, y) ;
$$

(A4) for each $x \in C, y \mapsto \Theta(x, y)$ is convex and lower semicontinuous;

(A5) for each $y \in C, x \mapsto \Theta(x, y)$ is weakly upper semicontinuous;

(B1) for each $x \in H$ and $r>0$, there exist a bounded subset $D_{x} \subseteq C$ and $y_{x} \in C$ such that for any $z \in C \backslash D_{x}$,

$$
\Theta\left(z, y_{x}\right)+\varphi\left(y_{x}\right)-\varphi(z)+\frac{1}{r}\left\langle y_{x}-z, z-x\right\rangle<0 ;
$$

(B2) $C$ is a bounded set.

The following lemmas were given in $[3,21]$. 
Lemma 2.1 [3] Let $C$ be a nonempty closed convex subset of $H$ and $\Theta$ be a bifunction of $C \times C$ into $\mathbb{R}$ satisfying (A1)-(A4). Let $r>0$ and $x \in H$. Then there exists $z \in C$ such that

$$
\Theta(z, y)+\frac{1}{r}\langle y-z, z-x\rangle \geq 0, \quad \forall y \in C .
$$

Lemma 2.2 [21] Let $C$ be a nonempty closed convex subset of $H$. Let $\Theta$ be a bifunction from $C \times C$ to $\mathbb{R}$ satisfying (A1)-(A5) and $\varphi: C \rightarrow \mathbb{R}$ be a proper lower semicontinuous and convex function. For $r>0$ and $x \in H$, define a mapping $T_{r}: H \rightarrow C$ as follows:

$$
T_{r} x=\left\{z \in C: \Theta(z, y)+\varphi(y)-\varphi(z)+\frac{1}{r}\langle y-z, z-x\rangle \geq 0, \forall y \in C\right\}
$$

for all $x \in H$. Assume that either (B1) or (B2) holds. Then the following hold:

(1) for each $x \in H, T_{r} x \neq \emptyset$;

(2) $T_{r}$ is single-valued;

(3) $T_{r}$ is firmly nonexpansive; that is, for any $x, y \in H$,

$$
\left\|T_{r} x-T_{r} y\right\|^{2} \leq\left\langle T_{r} x-T_{r} y, x-y\right\rangle
$$

(4) $F\left(T_{r}\right)=\operatorname{MEP}(\Theta, \varphi)$;

(5) $\operatorname{MEP}(\Theta, \varphi)$ is closed and convex.

We need the following lemmas for the proof of our main results.

Lemma 2.3 [22] Let $H$ be a Hilbert space, $C$ be a closed convex subset of $H$. If $S$ is a $k$ strictly pseudocontractive mapping on $C$, then the fixed point set $F(S)$ is closed convex, so that the projection $P_{F(S)}$ is well defined.

Lemma 2.4 [22] Let $H$ be a real Hilbert space and $C$ be a closed convex subset of $H$. Let $S: C \rightarrow H$ be a k-strictly pseudocontractive mapping with $F(S) \neq \emptyset$. Then $F\left(P_{C} S\right)=F(S)$.

Lemma 2.5 [22] Let $H$ be a real Hilbert space, $C$ be a closed convex subset of $H$, and $S: C \rightarrow H$ be a k-strictly pseudocontractive mapping. Define a mapping $T: C \rightarrow H$ by $T x=\lambda x+(1-\lambda) S x$ for all $x \in C$. Then as $\lambda \in[k, 1), T$ is a nonexpansive mapping such that $F(T)=F(S)$.

Lemma 2.6 [23] Let $\left\{s_{n}\right\}$ be a sequence of non-negative real numbers satisfying

$$
s_{n+1} \leq\left(1-\xi_{n}\right) s_{n}+\xi_{n} \delta_{n}, \quad \forall n \geq 1,
$$

where $\left\{\xi_{n}\right\}$ and $\left\{\delta_{n}\right\}$ satisfy the following conditions:

(i) $\left\{\xi_{n}\right\} \subset[0,1]$ and $\sum_{n=1}^{\infty} \xi_{n}=\infty$,

(ii) $\lim \sup _{n \rightarrow \infty} \delta_{n} \leq 0$ or $\sum_{n=1}^{\infty} \xi_{n} \delta_{n}<\infty$.

Then $\lim _{n \rightarrow \infty} s_{n}=0$.

Lemma 2.7 [24] Let $\left\{x_{n}\right\}$ and $\left\{z_{n}\right\}$ be bounded sequences in a real Banach space $E$ and $\left\{\gamma_{n}\right\}$ be a sequence in $[0,1]$ which satisfies the following condition:

$$
0<\liminf _{n \rightarrow \infty} \gamma_{n} \leq \limsup _{n \rightarrow \infty} \gamma_{n}<1 .
$$


Suppose that $x_{n+1}=\gamma_{n} x_{n}+\left(1-\gamma_{n}\right) z_{n}$ for all $n \geq 1$ and

$$
\limsup _{n \rightarrow \infty}\left(\left\|z_{n+1}-z_{n}\right\|-\left\|x_{n+1}-x_{n}\right\|\right) \leq 0
$$

Then $\lim _{n \rightarrow \infty}\left\|z_{n}-x_{n}\right\|=0$.

Lemma 2.8 In a real Hilbert space $H$, the following inequality holds:

$$
\|x+y\|^{2} \leq\|x\|^{2}+2\langle y, x+y\rangle, \quad \forall x, y \in H
$$

The following lemma can be easily proven, and therefore, we omit the proof.

Lemma 2.9 Let $V: H \rightarrow H$ be an l-Lipschitzian mapping with a constant $l \geq 0$, and $F$ : $H \rightarrow H$ be a $\rho$-Lipschitzian and $\eta$-strongly monotone operator with constants $\rho, \eta>0$. Then for $0 \leq \gamma l<\mu \eta$,

$$
\langle(\mu F-\gamma V) x-(\mu F-\gamma V) y, x-y\rangle \geq(\mu \eta-\gamma l)\|x-y\|^{2}, \quad \forall x, y \in C .
$$

That is, $\mu F-\gamma V$ is strongly monotone with a constant $\mu \eta-\gamma l$.

Finally, the following lemma is an improvement of Lemma 2.9 in [20] (see also [15]).

Lemma 2.10 Let $H$ be a real Hilbert space $H$. Let $F: H \rightarrow H$ be a $\rho$-Lipschizian and $\eta$-strongly monotone operator with $0<\eta \leq \rho$. Let $0<\mu<\frac{2 \eta}{\rho^{2}}$ and $0<t<\varsigma \leq 1$. Then $S:=\varsigma I-t \mu F: H \rightarrow H$ is a contraction with a contractive constant $\varsigma-t \tau$, where $\tau=$ $1-\sqrt{1-\mu\left(2 \eta-\mu \rho^{2}\right)}$.

Proof First, we show that $I-\mu F$ is strictly contractive. In fact, by applying the $\rho$-Lipschitz continuity and $\eta$-strongly monotonicity of $F$, we obtain for $x, y \in H$,

$$
\begin{aligned}
\|(I-\mu F) x-(I-\mu F) y\|^{2} \\
\quad=\|(x-y)-\mu(F x-F y)\|^{2} \\
\quad=\|x-y\|^{2}-2 \mu\langle F x-F y, x-y\rangle+\mu^{2}\|F x-F y\|^{2} \\
\quad \leq\|x-y\|^{2}-2 \mu \eta\|x-y\|^{2}+\mu^{2} \rho^{2}\|x-y\|^{2} \\
\quad=\left(1-\mu\left(2 \eta-\mu \rho^{2}\right)\right)\|x-y\|^{2},
\end{aligned}
$$

and so

$$
\|(I-\mu F) x-(I-\mu F) y\| \leq \sqrt{1-\mu\left(2 \eta-\mu \rho^{2}\right)}\|x-y\| .
$$

Now, noting that $S:=\varsigma I-t \mu F=(\varsigma-t) I-t(\mu F-I)$, by (2.3) we have for $x, y \in H$,

$$
\begin{aligned}
\|S x-S y\| & =\|(\varsigma-t)(x-y)-t((\mu F-I) x-(\mu F-I) y)\| \\
& \leq(\varsigma-t)\|x-y\|+t\|(\mu F-I) x-(\mu F-I) y\|
\end{aligned}
$$




$$
\begin{aligned}
& \leq(\varsigma-t)\|x-y\|+t \sqrt{1-\mu\left(2 \eta-\mu \rho^{2}\right)}\|x-y\| \\
& =\left(\varsigma-t\left(1-\sqrt{1-\mu\left(2 \eta-\mu \rho^{2}\right)}\right)\right)\|x-y\| \\
& =(\varsigma-t \tau)\|x-y\| .
\end{aligned}
$$

Hence, $S$ is a contraction with a contractive constant $\varsigma-t \tau$.

\section{Main results}

Let $H$ be a real Hilbert space, and let $C$ be a nonempty closed convex subset of $H$. Let $V: H \rightarrow H$ be an $l$-Lipschitzian mapping with a constant $l>0$, and $F: H \rightarrow H$ be a $\rho$ Lipschitzian and $\eta$-strongly monotone operator with $0<\eta \leq \rho$. Let $0<\mu<\frac{2 \eta}{\rho^{2}}$ and $0<$ $\gamma l<\tau$, where $\tau=1-\sqrt{1-\mu\left(2 \eta-\mu \rho^{2}\right)}$. Let $\left\{T_{r_{n}}\right\}$ be a sequence of mappings defined as in Lemma 2.2 and $S: C \rightarrow H$ be a $k$-strictly pseudocontractive mapping. Define a mapping $S_{n}: C \rightarrow H$ by $S_{n} x=\beta_{n} x+\left(1-\beta_{n}\right) S x, \forall x \in C$, where $\beta_{n} \in[k, 1)$. Then, by Lemma $2.5, S_{n}$ is nonexpansive.

Consider the following mapping $Q_{n}$ on $H$ defined by

$$
Q_{n} x=\alpha_{n} \gamma V x+\left(I-\alpha_{n} \mu F\right) S_{n} T_{r_{n}} x, \quad \forall x \in H, n \geq 1,
$$

where $\alpha_{n} \in(0,1)$. By Lemmas 2.2 and 2.10, we have

$$
\begin{aligned}
\left\|Q_{n} x-Q_{n} y\right\| & \leq \alpha_{n} \gamma\|V x-V y\|+\left\|\left(I-\alpha_{n} \mu F\right) S_{n} T_{r_{n}} x-\left(I-\alpha_{n} \mu F\right) S_{n} T_{r_{n}} y\right\| \\
& \leq \alpha_{n} \gamma l\|x-y\|+\left(1-\alpha_{n} \tau\right)\|x-y\| \\
& =\left(1-\alpha_{n}(\tau-\gamma l)\right)\|x-y\| .
\end{aligned}
$$

Since $0<1-\alpha_{n}(\tau-\gamma l)<1, Q_{n}$ is a contraction. Therefore, by the Banach contraction principle, $Q_{n}$ has a unique fixed point $x_{n} \in H$, which uniquely solves the fixed point equation

$$
x_{n}=\alpha_{n} \gamma V x_{n}+\left(I-\alpha_{n} \mu F\right) S_{n} T_{r_{n}} x_{n} .
$$

Now, we prove the convergence of the sequence $\left\{x_{n}\right\}$ and show the existence of the $q \in$ $\operatorname{MEP}(\Theta, \varphi) \cap F(S)$, which solves the variational inequality

$$
\langle(\mu F-\gamma V) q, p-q\rangle \geq 0, \quad \forall p \in \operatorname{MEP}(\Theta, \varphi) \cap F(S) .
$$

Equivalently, $q=P_{\operatorname{MEP}(\Theta, \varphi) \cap F(S)}(I-\mu F+\gamma V) q$.

Theorem 3.1 Let $C$ be a nonempty closed convex subset of a real Hilbert space $H$ and $\Theta$ be a bifunction from $C \times C \rightarrow \mathbb{R}$ satisfying (A1)-(A5). Let $S: C \rightarrow H$ be a k-strictly pseudocontractive non-self mapping such that $F(S) \cap \operatorname{MEP}(\Theta, \varphi) \neq \emptyset$. Let $F: H \rightarrow H$ be a $\rho$-Lipschitzian and $\eta$-strongly monotone operator with $0<\eta \leq \rho$. Let $V: H \rightarrow H$ be an l-Lipschitzian mapping with a constant $l>0$. Let $0<\mu<\frac{2 \eta}{\rho^{2}}$ and $0<\gamma l<\tau$, where $\tau=1-\sqrt{1-\mu\left(2 \eta-\mu \rho^{2}\right)}$. Assume that either (B1) or (B2) holds. Let $\left\{x_{n}\right\}$ be a sequence 
generated by

$$
\left\{\begin{array}{l}
\Theta\left(u_{n}, y\right)+\varphi(y)-\varphi\left(u_{n}\right)+\frac{1}{r_{n}}\left\langle y-u_{n}, u_{n}-x_{n}\right\rangle \geq 0, \quad \forall y \in C, \\
y_{n}=\beta_{n} u_{n}+\left(1-\beta_{n}\right) S u_{n}, \\
x_{n}=\alpha_{n} \gamma V x_{n}+\left(I-\alpha_{n} \mu F\right) y_{n}, \quad \forall n \geq 1,
\end{array}\right.
$$

where $u_{n}=T_{r_{n}} x_{n}, y_{n}=S_{n} u_{n}$, and $\left\{r_{n}\right\} \subset(0, \infty)$ satisfying $\liminf _{n \rightarrow \infty} r_{n}>0$. If $\left\{\alpha_{n}\right\}$ and $\left\{\beta_{n}\right\}$ satisfy the following conditions:

(i) $\left\{\alpha_{n}\right\} \subset(0,1), \lim _{n \rightarrow \infty} \alpha_{n}=0$;

(ii) $0 \leq k \leq \beta_{n} \leq \lambda<1$ and $\lim _{n \rightarrow \infty} \beta_{n}=\lambda$,

then $\left\{x_{n}\right\}$ converges strongly to a point $q \in F(S) \cap \operatorname{MEP}(\Theta, \varphi)$, which solves the variational inequality (3.1).

Proof Note that from the condition (i), without loss of generality, we assume that $\alpha_{n} \tau<1$ for $n \geq 1$.

First, we can show easily the uniqueness of a solution of the variational inequality (3.1). In fact, noting that $0 \leq \gamma l<\tau$ and $\mu \eta \geq \tau \Leftrightarrow \rho \geq \eta$, it follows from Lemma 2.9 that

$$
\langle(\mu F-\gamma V) x-(\mu F-\gamma V) y, x-y\rangle \geq(\mu \eta-\gamma l)\|x-y\|^{2} .
$$

That is, $\mu F-\gamma V$ is strongly monotone for $0 \leq \gamma l<\tau \leq \mu \eta$. So, the variational inequality (3.1) has only one solution. In what follows, we use $q \in F(S) \cap \operatorname{MEP}(\Theta, \varphi)$ to denote the unique solution of the variational inequality (3.1).

Now, take $p \in F(S) \cap \operatorname{MEP}(\Theta, \varphi)$. Since $u_{n}=T_{r_{n}} x_{n}$ and $p=T_{r_{n}} p$, from Lemma 2.2, we know that

$$
\left\|u_{n}-p\right\| \leq\left\|x_{n}-p\right\|, \quad \forall n \geq 1
$$

Moreover, from $S_{n} p=p$, it follows that

$$
\left\|y_{n}-p\right\|=\left\|S_{n} u_{n}-S_{n} p\right\| \leq\left\|u_{n}-p\right\| \leq\left\|x_{n}-p\right\|
$$

Thus, we have

$$
\begin{aligned}
\left\|x_{n}-p\right\| & =\left\|\alpha_{n}\left(\gamma V x_{n}-\mu F p\right)+\left(I-\alpha_{n} \mu F\right) y_{n}-\left(I-\alpha_{n} \mu F\right) p\right\| \\
& \leq\left(1-\alpha_{n} \tau\right)\left\|y_{n}-p\right\|+\alpha_{n}\left(\gamma l\left\|x_{n}-p\right\|+\|\gamma V p-\mu F p\|\right) \\
& \leq\left(1-\alpha_{n}(\tau-\gamma l)\right)\left\|x_{n}-p\right\|+\alpha_{n}\|\gamma V p-\mu F p\| .
\end{aligned}
$$

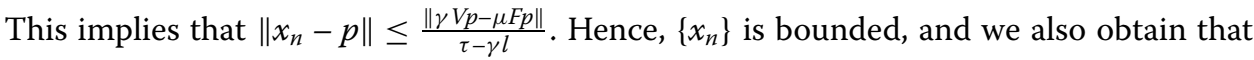
$\left\{u_{n}\right\},\left\{y_{n}\right\}$ and $\left\{V x_{n}\right\}$ are bounded. We note that

$$
\begin{aligned}
\left\|u_{n}-y_{n}\right\| & \leq\left\|u_{n}-x_{n}\right\|+\left\|x_{n}-y_{n}\right\| \\
& =\left\|u_{n}-x_{n}\right\|+\alpha_{n}\left\|\gamma V x_{n}-\mu F y_{n}\right\| .
\end{aligned}
$$


Using Lemma 2.2, we obtain

$$
\begin{aligned}
\left\|u_{n}-p\right\|^{2} & =\left\|T_{r_{n}} x_{n}-T_{r_{n}} p\right\|^{2} \\
& \leq\left\langle x_{n}-p, u_{n}-p\right\rangle=\frac{1}{2}\left(\left\|x_{n}-p\right\|^{2}+\left\|u_{n}-p\right\|^{2}-\left\|x_{n}-u_{n}\right\|^{2}\right),
\end{aligned}
$$

and so

$$
\left\|u_{n}-p\right\|^{2} \leq\left\|x_{n}-p\right\|^{2}-\left\|x_{n}-u_{n}\right\|^{2}
$$

Then, from Lemma 2.8, (3.3) and (3.5), we have

$$
\begin{aligned}
\left\|x_{n}-p\right\|^{2}= & \left\|\alpha_{n}\left(\gamma V x_{n}-\mu F p\right)+\left(I-\alpha_{n} \mu F\right) y_{n}-\left(I-\alpha_{n} \mu F\right) p\right\|^{2} \\
\leq & \left(1-\alpha_{n} \tau\right)^{2}\left\|y_{n}-p\right\|^{2}+2 \alpha_{n}\left\langle\gamma V x_{n}-\mu F p, x_{n}-p\right\rangle \\
\leq & \left(1-\alpha_{n} \tau\right)^{2}\left\|u_{n}-p\right\|^{2}+2 \alpha_{n} \gamma\left\langle V x_{n}-V p, x_{n}-p\right\rangle \\
& +2 \alpha_{n}\|\gamma V p-\mu F p\|\left\|x_{n}-p\right\| \\
\leq & \left(1-\alpha_{n} \tau\right)^{2}\left(\left\|x_{n}-p\right\|^{2}-\left\|x_{n}-u_{n}\right\|^{2}\right)+2 \alpha_{n} \gamma l\left\|x_{n}-p\right\|^{2} \\
& +2 \alpha_{n}\|\gamma V p-\mu F p\|\left\|x_{n}-p\right\| \\
= & \left(1-2 \alpha_{n}(\tau-\gamma l)+\left(\alpha_{n} \tau\right)^{2}\right)\left\|x_{n}-p\right\|^{2}-\left(1-\alpha_{n} \tau\right)^{2}\left\|x_{n}-u_{n}\right\|^{2} \\
& +2 \alpha_{n}\|\gamma V p-\mu F p\|\left\|x_{n}-p\right\| \\
\leq & \left\|x_{n}-p\right\|^{2}+\alpha_{n}^{2} \tau^{2}\left\|x_{n}-p\right\|^{2}-\left(1-\alpha_{n} \tau\right)^{2}\left\|x_{n}-u_{n}\right\|^{2} \\
& +2 \alpha_{n}\|\gamma V p-\mu F p\|\left\|x_{n}-p\right\|,
\end{aligned}
$$

and hence

$$
\left(1-\alpha_{n} \tau\right)^{2}\left\|x_{n}-u_{n}\right\|^{2} \leq \alpha_{n}^{2} \tau^{2}\left\|x_{n}-p\right\|^{2}+2 \alpha_{n}\|\gamma V p-\mu F p\|\left\|x_{n}-p\right\| .
$$

Since $\alpha_{n} \rightarrow 0$, it follows that

$$
\lim _{n \rightarrow \infty}\left\|x_{n}-u_{n}\right\|=0
$$

From (3.4), we know that

$$
\lim _{n \rightarrow \infty}\left\|u_{n}-y_{n}\right\|=0
$$

Define $T: C \rightarrow H$ by $T x=\lambda x+(1-\lambda) S x$. Then by Lemma $2.5, T$ is nonexpansive with $F(T)=F(S)$. Notice that

$$
\begin{aligned}
\left\|T u_{n}-u_{n}\right\| & \leq\left\|T u_{n}-y_{n}\right\|+\left\|y_{n}-u_{n}\right\| \\
& \leq\left|\lambda-\beta_{n}\right|\left\|u_{n}-S u_{n}\right\|+\left\|y_{n}-u_{n}\right\| .
\end{aligned}
$$

By (3.6) and $\beta_{n} \rightarrow \lambda$, we obtain

$$
\lim _{n \rightarrow \infty}\left\|T u_{n}-u_{n}\right\|=0
$$


Consider a subsequence $\left\{u_{n_{i}}\right\}$ of $\left\{u_{n}\right\}$. Since $\left\{u_{n}\right\}$ is bounded, there exists a subsequence $\left\{u_{n_{i}}\right\}$ of $\left\{u_{n_{i}}\right\}$ which converges weakly to $q$.

Next, we show that $q \in F(S) \cap \operatorname{MEP}(\Theta, \varphi)$. Without loss of generality, we can assume that $u_{n_{i}} \rightarrow q$. Since $C$ is closed and convex, $C$ is weakly closed. So, we have $q \in C$. Let us show $q \in F(T)$. Assume that $q \notin F(T)$. Since $u_{n_{i}} \rightarrow q$ and $q \neq T q$, it follows from the Opial condition that

$$
\begin{aligned}
\liminf _{i \rightarrow \infty}\left\|u_{n_{i}}-q\right\| & <\liminf _{i \rightarrow \infty}\left\|u_{n_{i}}-T q\right\| \\
& \leq \liminf _{i \rightarrow \infty}\left(\left\|u_{n_{i}}-T u_{n_{i}}\right\|+\left\|T u_{n_{i}}-T q\right\|\right) \\
& \leq \liminf _{i \rightarrow \infty}\left\|u_{n_{i}}-q\right\|,
\end{aligned}
$$

which is a contradiction. So, we get $q \in F(T)$, and hence $q \in F(S)$.

We shall show that $q \in \operatorname{MEP}(\Theta, \varphi)$. Since $u_{n}=T_{r_{n}} x_{n}$, for any $y \in C$, we have

$$
\Theta\left(u_{n}, y\right)+\varphi(y)-\varphi\left(u_{n}\right)+\frac{1}{r_{n}}\left\langle y-u_{n}, u_{n}-x_{n}\right\rangle \geq 0 .
$$

It follows from (A2) that

$$
\varphi(y)-\varphi\left(u_{n}\right)+\frac{1}{r_{n}}\left\langle y-u_{n}, u_{n}-x_{n}\right\rangle \geq \Theta\left(y, u_{n}\right) .
$$

Replacing $n$ by $n_{i}$, we have

$$
\varphi(y)-\varphi\left(u_{n_{i}}\right)+\frac{1}{r_{n_{i}}}\left\langle y-u_{n_{i}}, u_{n_{i}}-x_{n_{i}}\right\rangle \geq \Theta\left(y, u_{n_{i}}\right) .
$$

Since $\frac{u_{n_{i}}-x_{n_{i}}}{r_{n_{i}}} \rightarrow 0$ and $u_{n_{i}} \rightarrow q$, it follows from (A4) that

$$
0 \geq-\varphi(y)+\varphi(q)+\Theta(y, q), \quad \forall y \in C
$$

Put $z_{t}=t y+(1-t) q$ for all $t \in(0,1]$ and $y \in C$. Then we have $z_{t} \in C$ and

$$
-\varphi\left(z_{t}\right)+\varphi(q)+\Theta\left(z_{t}, q\right) \leq 0 .
$$

By (A1), (A4) and (3.7), we have

$$
\begin{aligned}
0 & =\Theta\left(z_{t}, z_{t}\right)+\varphi\left(z_{t}\right)-\varphi\left(z_{t}\right) \\
& \leq t \Theta\left(z_{t}, y\right)+(1-t) \Theta\left(z_{t}, q\right)+t \varphi(y)+(1-t) \varphi(q)-\varphi\left(z_{t}\right) \\
& \leq t\left(\Theta\left(z_{t}, y\right)+\varphi(y)-\varphi\left(z_{t}\right)\right)+(1-t)\left(\Theta\left(z_{t}, q\right)+\varphi(q)-\varphi\left(z_{t}\right)\right) \\
& \leq t\left(\Theta\left(z_{t}, y\right)+\varphi(y)-\varphi\left(z_{t}\right)\right),
\end{aligned}
$$

and hence

$$
0 \leq \Theta\left(z_{t}, y\right)+\varphi(y)-\varphi\left(z_{t}\right) .
$$


Letting $t \rightarrow 0$, by (A3) we have for each $y \in C$,

$$
0 \leq \Theta(q, y)+\varphi(y)-\varphi(q) .
$$

This implies that $q \in \operatorname{MEP}(\Theta, \varphi)$. Therefore, $q \in F(S) \cap \operatorname{MEP}(\Theta, \varphi)$.

On the other hand, we note that

$$
x_{n}-q=\alpha_{n}\left(\gamma V x_{n}-\mu F q\right)+\left(I-\alpha_{n} \mu F\right) y_{n}-\left(I-\alpha_{n} \mu F\right) q .
$$

It follows that

$$
\begin{aligned}
\| x_{n} & -q \|^{2} \\
& =\alpha_{n}\left\langle\gamma V x_{n}-\mu F q, x_{n}-q\right\rangle+\left\langle\left(I-\alpha_{n} \mu F\right) y_{n}-\left(I-\alpha_{n} \mu F\right) q, x_{n}-q\right\rangle \\
& \leq \alpha_{n}\left\langle\gamma V x_{n}-\mu F q, x_{n}-q\right\rangle+\left\|\left(I-\alpha_{n} \mu F\right) y_{n}-\left(I-\alpha_{n} \mu F\right) q\right\|\left\|x_{n}-q\right\| \\
& \leq \alpha_{n}\left\langle\gamma V x_{n}-\mu F q, x_{n}-q\right\rangle+\left(1-\alpha_{n} \tau\right)\left\|y_{n}-q\right\|\left\|x_{n}-q\right\| \\
& \leq \alpha_{n}\left\langle\gamma V x_{n}-\mu F q, x_{n}-q\right\rangle+\left(1-\alpha_{n} \tau\right)\left\|x_{n}-q\right\|^{2} .
\end{aligned}
$$

Hence, we obtain

$$
\begin{aligned}
\left\|x_{n}-q\right\|^{2} & \leq \frac{1}{\tau}\left\langle\gamma V x_{n}-\mu F q, x_{n}-q\right\rangle \\
& =\frac{1}{\tau}\left(\gamma\left\langle V x_{n}-V q, x_{n}-q\right\rangle+\left\langle\gamma V q-\mu F q, x_{n}-q\right\rangle\right) \\
& \leq \frac{1}{\tau}\left(\gamma l\left\|x_{n}-q\right\|^{2}+\left\langle\gamma V q-\mu F q, x_{n}-q\right\rangle\right) .
\end{aligned}
$$

This implies that

$$
\left\|x_{n}-q\right\|^{2} \leq \frac{\left\langle\gamma V q-\mu F q, x_{n}-q\right\rangle}{\tau-\gamma l} .
$$

In particular, we have

$$
\left\|x_{n_{i}}-q\right\|^{2} \leq \frac{\left\langle\gamma V q-\mu F q, x_{n_{i}}-q\right\rangle}{\tau-\gamma l} .
$$

Since $x_{n_{i}} \rightarrow q$, it follows that $x_{n_{i}} \rightarrow q$ as $i \rightarrow \infty$.

Now, we show that $q$ solves the variational inequality (3.1). Since $x_{n}=\alpha_{n} \gamma V x_{n}+(I-$ $\left.\alpha_{n} \mu F\right) S_{n} T_{r_{n}} x_{n}$, we have

$$
(\mu F-\gamma V) x_{n}=-\frac{1}{\alpha_{n}}\left(\left(I-\alpha_{n} \mu F\right) x_{n}-\left(I-\alpha_{n} \mu F\right) S_{n} T_{r_{n}} x_{n}\right) .
$$

It follows that for $p \in F(S) \cap \operatorname{MEP}(\Theta, \varphi)$,

$$
\begin{aligned}
\left\langle(\mu F-\gamma V) x_{n}, x_{n}-p\right\rangle & =-\frac{1}{\alpha_{n}}\left\langle\left(I-\alpha_{n} \mu F\right) x_{n}-\left(I-\alpha_{n} \mu F\right) S_{n} T_{r_{n}} x_{n}, x_{n}-p\right\rangle \\
& =-\frac{1}{\alpha_{n}}\left\langle\left(I-S_{n} T_{r_{n}}\right) x_{n}-\left(I-S_{n} T_{r_{n}}\right) p, x_{n}-p\right\rangle
\end{aligned}
$$




$$
\begin{aligned}
& +\left\langle\mu F x_{n}-\mu F S_{n} T_{r_{n}} x_{n}, x_{n}-p\right\rangle \\
\leq & \left\langle\mu F x_{n}-\mu F S_{n} T_{r_{n}} x_{n}, x_{n}-p\right\rangle
\end{aligned}
$$

since $I-S_{n} T_{r_{n}}$ is monotone (i.e., $\left\langle x-y,\left(I-S_{n} T_{r_{n}}\right) x-\left(I-S_{n} T_{r_{n}}\right) y\right\rangle \geq 0$ for all $x, y \in H$. This is due to the nonexpansivity of $\left.S_{n} T_{r_{n}}\right)$. Since $\left\|x_{n}-y_{n}\right\|=\alpha_{n}\left\|\gamma V x_{n}-\mu F y_{n}\right\| \rightarrow 0$ as $n \rightarrow \infty$, by replacing $n$ in (3.10) with $n_{i}$ and letting $i \rightarrow \infty$, we obtain

$$
\begin{aligned}
\langle(\mu F-\gamma V) q, q-p\rangle & =\lim _{i \rightarrow \infty}\left\langle(\mu F-\gamma V) x_{n_{i}}, x_{n_{i}}-p\right\rangle \\
& \leq \lim _{i \rightarrow \infty}\left\langle\mu F x_{n_{i}}-\mu F y_{n_{i}}, x_{n_{i}}-p\right\rangle=0 .
\end{aligned}
$$

That is, $q \in F(S) \cap \operatorname{MEP}(\Theta, \varphi)$ is a solution of the variational inequality (3.1).

Finally, we show that the sequence $\left\{x_{n}\right\}$ converges strongly to $q$. To this end, let $\left\{x_{n_{k}}\right\}$ be another subsequence of $\left\{x_{n}\right\}$ and assume $x_{n_{k}} \rightarrow \widehat{q}$. By the same proof as the one above, we have $\widehat{q} \in F(S) \cap \operatorname{MEP}(\Theta, \varphi)$. Moreover, it follows from (3.10) that

$$
\langle(\mu F-\gamma V) q, q-\widehat{q}\rangle \leq 0
$$

Interchanging $q$ and $\widehat{q}$, we obtain

$$
\langle(\mu F-\gamma V) \widehat{q}, \widehat{q}-q\rangle \leq 0
$$

Lemma 2.9 and adding these two inequalities (3.12) and (3.13) yield

$$
(\mu \eta-\gamma l)\|q-\widehat{q}\|^{2} \leq\langle(\mu F-\gamma V) q-(\mu F-\gamma V) \widehat{q}, q-\widehat{q}\rangle \leq 0 .
$$

Hence, $q=\widehat{q}$. Therefore, we conclude that $x_{n} \rightarrow q$ as $n \rightarrow \infty$.

The variational inequality (3.1) can be rewritten as

$$
\langle(I-\mu F+\gamma V) q-q, q-p\rangle \geq 0, \quad \forall p \in F(S) \cap \operatorname{MEP}(\Theta, \varphi) .
$$

By (2.2), this is equivalent to the fixed point equation

$$
P_{F(S) \cap \operatorname{MEP}(\Theta, \varphi)}(I-\mu F+\gamma V) q=q .
$$

Now, we establish the strong convergence of an explicit iterative scheme for finding a common element of the set of solutions of a mixed equilibrium problem and the set of fixed points of a $k$-strictly pseudocontractive non-self mapping.

Theorem 3.2 Let $C$ be a nonempty closed convex subset of a real Hilbert space $H$ and $\Theta$ be a bifunction from $C \times C \rightarrow \mathbb{R}$ satisfying (A1)-(A5). Let $S: C \rightarrow H$ be a $k$-strictly pseudocontractive non-self mapping such that $F(S) \cap \operatorname{MEP}(\Theta, \varphi) \neq \emptyset$. Let $F: H \rightarrow H$ be a $\rho$-Lipschitzian and $\eta$-strongly monotone operator with $0<\eta \leq \rho$. Let $V: H \rightarrow H$ be an l-Lipschitzian mapping with a constant $l>0$. Let $0<\mu<\frac{2 \eta}{\rho^{2}}$ and $0<\gamma l<\tau$, where $\tau=$ $1-\sqrt{1-\mu\left(2 \eta-\mu \rho^{2}\right)}$. Assume that either (B1) or (B2) holds. Let $\left\{x_{n}\right\}$ and $\left\{u_{n}\right\}$ be sequences 
generated by

$$
\left\{\begin{array}{l}
\Theta\left(u_{n}, y\right)+\varphi(y)-\varphi\left(u_{n}\right)+\frac{1}{r_{n}}\left\langle y-u_{n}, u_{n}-x_{n}\right\rangle \geq 0, \quad \forall y \in C, \\
y_{n}=\beta_{n} u_{n}+\left(1-\beta_{n}\right) S u_{n}, \\
x_{n+1}=\alpha_{n} \gamma V x_{n}+\lambda_{n} x_{n}+\left(\left(1-\lambda_{n}\right) I-\alpha_{n} \mu F\right) y_{n}, \quad \forall n \geq 1,
\end{array}\right.
$$

where $u_{n}=T_{r_{n}} x_{n}$ and $y_{n}=S_{n} u_{n}$. If $\left\{\alpha_{n}\right\},\left\{\beta_{n}\right\},\left\{r_{n}\right\}$ and $\left\{\lambda_{n}\right\}$ satisfy the following conditions:

(i) $\left\{\alpha_{n}\right\} \subset(0,1), \lim _{n \rightarrow \infty} \alpha_{n}=0, \sum_{n=1}^{\infty} \alpha_{n}=\infty$;

(ii) $0 \leq k \leq \beta_{n} \leq \lambda<1$ and $\lim _{n \rightarrow \infty} \beta_{n}=\lambda$, $\lim _{n \rightarrow \infty}\left|\beta_{n+1}-\beta_{n}\right|=0$;

(iii) $\left\{r_{n}\right\} \subset(0, \infty), \liminf _{n \rightarrow \infty} r_{n}>0, \lim _{n \rightarrow \infty}\left|r_{n+1}-r_{n}\right|=0$;

(iv) $\left\{\lambda_{n}\right\} \subset(0,1)$ and $0<\liminf _{n \rightarrow \infty} \lambda_{n} \leq \limsup _{n \rightarrow \infty} \lambda_{n}<1$,

then $\left\{x_{n}\right\}$ and $\left\{u_{n}\right\}$ converge strongly to a point $q \in F(S) \cap \operatorname{MEP}(\Theta, \varphi)$, which solves the variational inequality (3.1).

Proof First, from the condition (i), without loss of generality, we assume that $\alpha_{n} \tau<1$, $\frac{2 \alpha_{n}(\tau-\gamma l)}{1-\alpha_{n} \gamma l}<1$ and $\alpha_{n}\left(1-\lambda_{n}\right)<1$ for $n \geq 1$.

We divide the proof into several steps as follows.

Step 1. We show that $\left\|x_{n}-p\right\| \leq \max \left\{\left\|x_{0}-p\right\|, \frac{\|\gamma V p-\mu F p\|}{\tau-\gamma l}\right\}$ for all $n \geq 0$ and all $p \in F(S) \cap$ $\operatorname{MEP}(\Theta, \varphi)$. Indeed, let $p \in F(S) \cap \operatorname{MEP}(\Theta, \varphi)$. Then from Lemma 2.10, we have

$$
\begin{aligned}
& \left\|x_{n+1}-p\right\| \\
& \quad=\left\|\alpha_{n}\left(\gamma V x_{n}-\mu F p\right)+\lambda_{n}\left(x_{n}-p\right)+\left(\left(1-\lambda_{n}\right) I-\alpha_{n} \mu F\right) y_{n}-\left(\left(1-\lambda_{n}\right) I-\alpha_{n} \mu F\right) p\right\| \\
& \quad \leq\left(1-\lambda_{n}-\alpha_{n} \tau\right)\left\|y_{n}-p\right\|+\lambda_{n}\left\|x_{n}-p\right\|+\alpha_{n}\left\|\gamma V x_{n}-\mu F p\right\| \\
& \quad \leq\left(1-\lambda_{n}-\alpha_{n} \tau\right)\left\|x_{n}-p\right\|+\lambda_{n}\left\|x_{n}-p\right\|+\alpha_{n}\left(\left\|\gamma V x_{n}-\gamma V p\right\|+\|\gamma V p-\mu F p\|\right) \\
& \quad \leq\left(1-\alpha_{n} \tau\right)\left\|x_{n}-p\right\|+\alpha_{n} \gamma l\left\|x_{n}-p\right\|+\alpha_{n}\|\gamma V p-\mu F p\| \\
& \quad=\left(1-(\tau-\gamma l) \alpha_{n}\right)\left\|x_{n}-p\right\|+(\tau-\gamma l) \alpha_{n} \frac{\|\gamma V p-\mu F p\|}{\tau-\gamma l} \\
& \quad \leq \max \left\{\left\|x_{n}-p\right\|, \frac{\|\gamma V p-\mu F p\|}{\tau-\gamma l}\right\} .
\end{aligned}
$$

From induction, we have

$$
\left\|x_{n}-p\right\| \leq \max \left\{\left\|x_{1}-p\right\|, \frac{\|\gamma V p-\mu F p\|}{\tau-\gamma l}\right\}, \quad \forall n \geq 1 .
$$

Hence, $\left\{x_{n}\right\}$ is bounded. From (3.3), $\left\{u_{n}\right\},\left\{y_{n}\right\},\left\{V x_{n}\right\},\left\{S u_{n}\right\}$ and $\left\{F y_{n}\right\}$ are also bounded.

Step 2. We show that $\lim _{n \rightarrow \infty}\left\|x_{n+1}-x_{n}\right\|=0$ and $\lim _{n \rightarrow \infty}\left\|u_{n+1}-u_{n}\right\|=0$. To show this, define

$$
x_{n+1}=\lambda_{n} x_{n}+\left(1-\lambda_{n}\right) z_{n}, \quad \forall n \geq 1 .
$$

Observe that from the definition of $z_{n}$,

$$
\begin{aligned}
z_{n+1}-z_{n} & =\frac{x_{n+2}-\lambda_{n+1} x_{n+1}}{1-\lambda_{n+1}}-\frac{x_{n+1}-\lambda_{n} x_{n}}{1-\lambda_{n}} \\
& =\frac{\alpha_{n+1} \gamma V x_{n+1}+\left(\left(1-\lambda_{n+1}\right) I-\alpha_{n+1} \mu F\right) y_{n+1}}{1-\lambda_{n+1}}
\end{aligned}
$$




$$
\begin{aligned}
& -\frac{\alpha_{n} \gamma V x_{n}+\left(\left(1-\lambda_{n}\right) I-\alpha_{n} \mu F\right) y_{n}}{1-\lambda_{n}} \\
= & \frac{\alpha_{n+1}}{1-\lambda_{n+1}} \gamma V x_{n+1}-\frac{\alpha_{n}}{1-\lambda_{n}} \gamma V x_{n} \\
& +y_{n+1}-y_{n}+\frac{\alpha_{n}}{1-\lambda_{n}} \mu F y_{n}-\frac{\alpha_{n+1}}{1-\lambda_{n+1}} \mu F y_{n+1} \\
= & \frac{\alpha_{n+1}}{1-\lambda_{n+1}}\left(\gamma V x_{n+1}-\mu F y_{n+1}\right)+\frac{\alpha_{n}}{1-\lambda_{n}}\left(\mu F y_{n}-\gamma V x_{n}\right)+y_{n+1}-y_{n} .
\end{aligned}
$$

Thus, it follows that

$$
\begin{aligned}
\left\|z_{n+1}-z_{n}\right\| \leq & \frac{\alpha_{n+1}}{1-\lambda_{n+1}}\left(\gamma\left\|V x_{n+1}\right\|+\mu\left\|F y_{n+1}\right\|\right) \\
& +\frac{\alpha_{n}}{1-\lambda_{n}}\left(\mu\left\|F y_{n}\right\|+\gamma\left\|V x_{n}\right\|\right)+\left\|y_{n+1}-y_{n}\right\| .
\end{aligned}
$$

On the one hand, we note that

$$
\begin{aligned}
\left\|y_{n+1}-y_{n}\right\| & =\left\|S_{n+1} u_{n+1}-S_{n} u_{n}\right\| \\
& \leq\left\|S_{n+1} u_{n+1}-S_{n+1} u_{n}\right\|+\left\|S_{n+1} u_{n}-S_{n} u_{n}\right\| \\
& \leq\left\|u_{n+1}-u_{n}\right\|+\left\|S_{n+1} u_{n}-S_{n} u_{n}\right\| .
\end{aligned}
$$

Noticing that

$$
\begin{aligned}
\left\|s_{n+1} u_{n}-S_{n} u_{n}\right\| & =\left\|\beta_{n+1} u_{n}+\left(1-\beta_{n+1}\right) S u_{n}-\left(\beta_{n} u_{n}+\left(1-\beta_{n}\right) S u_{n}\right)\right\| \\
& \leq\left|\beta_{n+1}-\beta_{n}\right|\left\|u_{n}-S u_{n}\right\|
\end{aligned}
$$

from (3.16) we have

$$
\left\|y_{n+1}-y_{n}\right\| \leq\left\|u_{n+1}-u_{n}\right\|+\left|\beta_{n+1}-\beta_{n}\right|\left\|u_{n}-S u_{n}\right\|
$$

On the other hand, from $u_{n+1}=T_{r_{n+1}} x_{n+1}$ and $u_{n}=T_{r_{n}} x_{n}$, we have

$$
\Theta\left(u_{n+1}, y\right)+\varphi(y)-\varphi\left(u_{n+1}\right)+\frac{1}{r_{n+1}}\left\langle y-u_{n+1}, u_{n+1}-x_{n+1}\right\rangle \geq 0, \quad \forall y \in C
$$

and

$$
\Theta\left(u_{n}, y\right)+\varphi(y)-\varphi\left(u_{n}\right)+\frac{1}{r_{n}}\left\langle y-u_{n}, u_{n}-x_{n}\right\rangle \geq 0, \quad \forall y \in C .
$$

Putting $y=u_{n}$ in (3.18) and $y=u_{n+1}$ in (3.19), we obtain

$$
\Theta\left(u_{n+1}, u_{n}\right)+\varphi\left(u_{n}\right)-\varphi\left(u_{n+1}\right)+\frac{1}{r_{n+1}}\left\langle u_{n}-u_{n+1}, u_{n+1}-x_{n+1}\right\rangle \geq 0
$$

and

$$
\Theta\left(u_{n}, u_{n+1}\right)+\varphi\left(u_{n+1}\right)-\varphi\left(u_{n}\right)+\frac{1}{r_{n}}\left\langle u_{n+1}-u_{n}, u_{n}-x_{n}\right\rangle \geq 0 .
$$


By (A2), we have

$$
\left\langle u_{n+1}-u_{n}, \frac{u_{n}-x_{n}}{r_{n}}-\frac{u_{n+1}-x_{n+1}}{r_{n+1}}\right\rangle \geq 0
$$

and hence

$$
\left\langle u_{n+1}-u_{n}, u_{n}-u_{n+1}+u_{n+1}-x_{n}-\frac{r_{n}}{r_{n+1}}\left(u_{n+1}-x_{n+1}\right)\right\rangle \geq 0 .
$$

Since $\liminf _{n \rightarrow \infty} r_{n}>0$, we assume that there exists a real number $c$ such that $r_{n}>c>0$ for all $n \geq 1$. Thus, we have

$$
\begin{aligned}
\left\|u_{n+1}-u_{n}\right\|^{2} & \leq\left\langle u_{n+1}-u_{n}, x_{n+1}-x_{n}+\left(1-\frac{r_{n}}{r_{n+1}}\right)\left(u_{n+1}-x_{n+1}\right)\right\rangle \\
& \leq\left\|u_{n+1}-u_{n}\right\|\left\{\left\|x_{n+1}-x_{n}\right\|+\left|1-\frac{r_{n}}{r_{n+1}}\right|\left\|u_{n+1}-x_{n+1}\right\|\right\},
\end{aligned}
$$

and hence

$$
\begin{aligned}
\left\|u_{n+1}-u_{n}\right\| & \leq\left\|x_{n+1}-x_{n}\right\|+\frac{1}{r_{n+1}}\left|r_{n+1}-r_{n}\right|\left\|u_{n+1}-x_{n+1}\right\| \\
& \leq\left\|x_{n+1}-x_{n}\right\|+\frac{1}{c}\left|r_{n+1}-r_{n}\right| L
\end{aligned}
$$

where $L=\sup \left\{\left\|u_{n}-x_{n}\right\|: n \geq 1\right\}$. Therefore, from (3.15), (3.17) and (3.20), we obtain

$$
\begin{aligned}
& \left\|z_{n+1}-z_{n}\right\|-\left\|x_{n+1}-x_{n}\right\| \\
& \leq \frac{\alpha_{n+1}}{1-\lambda_{n+1}}\left(\gamma\left\|V x_{n+1}\right\|+\mu\left\|F y_{n+1}\right\|\right)+\frac{\alpha_{n}}{1-\lambda_{n}}\left(\mu\left\|F y_{n}\right\|+\gamma\left\|V x_{n}\right\|\right) \\
& \quad+\left|\beta_{n+1}-\beta_{n}\right|\left\|u_{n}-S u_{n}\right\|+\frac{1}{c}\left|r_{n+1}-r_{n}\right| L \\
& \leq \frac{\alpha_{n+1}}{1-\lambda_{n+1}}\left(\gamma\left\|V x_{n+1}\right\|+\mu\left\|F y_{n+1}\right\|\right)+\frac{\alpha_{n}}{1-\lambda_{n}}\left(\mu\left\|F y_{n}\right\|+\gamma\left\|V x_{n}\right\|\right) \\
& \quad+\left(\left|\beta_{n+1}-\beta_{n}\right|+\left|r_{n+1}-r_{n}\right|\right) M_{1},
\end{aligned}
$$

where $M_{1}$ is an appropriate constant such that $M_{1}=\frac{L}{c}+\sup \left\{\left\|u_{n}-S u_{n}\right\|: n \geq 1\right\}$. Thus, from conditions (i)-(iv), it follows that

$$
\limsup _{n \rightarrow \infty}\left(\left\|z_{n+1}-z_{n}\right\|-\left\|x_{n+1}-x_{n}\right\|\right) \leq 0
$$

Hence, by Lemma 2.7, we have

$$
\lim _{n \rightarrow \infty}\left\|z_{n}-x_{n}\right\|=0
$$

Consequently,

$$
\lim _{n \rightarrow \infty}\left\|x_{n+1}-x_{n}\right\|=\lim _{n \rightarrow \infty}\left(1-\lambda_{n}\right)\left\|z_{n}-x_{n}\right\|=0,
$$


and by (3.17) and (3.20),

$$
\lim _{n \rightarrow \infty}\left\|u_{n+1}-u_{n}\right\|=0, \quad \text { and } \quad \lim _{n \rightarrow \infty}\left\|y_{n+1}-y_{n}\right\|=0 .
$$

Step 3. We show that $\lim _{n \rightarrow \infty}\left\|x_{n}-y_{n}\right\|=0$. Indeed, since

$$
x_{n+1}=\alpha_{n} \gamma V x_{n}+\lambda_{n} x_{n}+\left(\left(1-\lambda_{n}\right) I-\alpha_{n} \mu F\right) y_{n},
$$

we have

$$
\begin{aligned}
\left\|x_{n}-y_{n}\right\| & \leq\left\|x_{n}-x_{n+1}\right\|+\left\|x_{n+1}-y_{n}\right\| \\
& \leq\left\|x_{n}-x_{n+1}\right\|+\alpha_{n}\left\|\gamma V x_{n}-\mu F y_{n}\right\|+\lambda_{n}\left\|x_{n}-y_{n}\right\|,
\end{aligned}
$$

that is,

$$
\left\|x_{n}-y_{n}\right\| \leq \frac{1}{1-\lambda_{n}}\left\|x_{n}-x_{n+1}\right\|+\frac{\alpha_{n}}{1-\lambda_{n}}\left(\gamma\left\|V x_{n}\right\|+\mu\left\|F y_{n}\right\|\right) .
$$

So, from the conditions $\alpha_{n} \rightarrow 0$ and (iv) and Step 2, it follows that

$$
\lim _{n \rightarrow \infty}\left\|x_{n}-y_{n}\right\|=0
$$

Step 4. We show that $\lim _{n \rightarrow \infty}\left\|x_{n}-u_{n}\right\|=0$ and $\lim _{n \rightarrow \infty}\left\|u_{n}-y_{n}\right\|=0$. Indeed, since $T_{r_{n}}$ is firmly nonexpansive, for $p \in F(S) \cap \operatorname{MEP}(\Theta, \varphi)$, we have

$$
\begin{aligned}
\left\|u_{n}-p\right\|^{2} & =\left\|T_{r_{n}} x_{n}-T_{r_{n}} p\right\|^{2} \\
& \leq\left\langle x_{n}-p, u_{n}-p\right\rangle \\
& =\frac{1}{2}\left(\left\|x_{n}-p\right\|^{2}+\left\|u_{n}-p\right\|^{2}-\left\|x_{n}-u_{n}\right\|^{2}\right),
\end{aligned}
$$

and hence

$$
\left\|u_{n}-p\right\|^{2} \leq\left\|x_{n}-p\right\|^{2}-\left\|x_{n}-u_{n}\right\|^{2} .
$$

Then, by using the convexity of $\|\cdot\|^{2}$, we have from (3.14) and (3.21),

$$
\begin{aligned}
&\left\|x_{n+1}-q\right\|^{2} \\
&=\left\|\alpha_{n}\left(\gamma V x_{n}+(I-\mu F) y_{n}-p\right)+\left(1-\alpha_{n}\right)\left(y_{n}-p\right)+\lambda_{n}\left(x_{n}-y_{n}\right)\right\|^{2} \\
& \leq\left(\left\|\alpha_{n}\left(\gamma V x_{n}+(I-\mu F) y_{n}-p\right)+\left(1-\alpha_{n}\right)\left(y_{n}-p\right)\right\|+\lambda_{n}\left\|x_{n}-y_{n}\right\|\right)^{2} \\
& \leq\left\|\alpha_{n}\left(\gamma V x_{n}+(I-\mu F) y_{n}-p\right)+\left(1-\alpha_{n}\right)\left(y_{n}-p\right)\right\|^{2} \\
&+2 \lambda_{n}\left\|\alpha_{n}\left(\gamma V x_{n}+(I-\mu F) y_{n}-p\right)+\left(1-\alpha_{n}\right)\left(y_{n}-p\right)\right\|\left\|x_{n}-y_{n}\right\| \\
&+\lambda_{n}^{2}\left\|x_{n}-y_{n}\right\|^{2} \\
& \leq \alpha_{n}\left\|\gamma V x_{n}+(I-\mu F) y_{n}-p\right\|^{2}+\left(1-\alpha_{n}\right)\left\|y_{n}-p\right\|^{2} \\
&+\lambda_{n}\left\|x_{n}-y_{n}\right\|\left[2 \alpha_{n}\left(\left\|\gamma V x_{n}+(I-\mu F) y_{n}-p\right\|+\left(1-\alpha_{n}\right)\left\|y_{n}-p\right\|\right)\right.
\end{aligned}
$$




$$
\begin{aligned}
& \left.+\lambda_{n}\left\|x_{n}-y_{n}\right\|\right] \\
\leq & \alpha_{n}\left\|\gamma V x_{n}+(I-\mu F) y_{n}-p\right\|^{2}+\left(1-\alpha_{n}\right)\left\|u_{n}-p\right\|^{2}+M_{n} \\
\leq & \alpha_{n}\left\|\gamma V x_{n}+(I-\mu F) y_{n}-p\right\|^{2}+\left(1-\alpha_{n}\right)\left(\left\|x_{n}-p\right\|^{2}-\left\|x_{n}-u_{n}\right\|^{2}\right)+M_{n},
\end{aligned}
$$

where

$$
M_{n}=\lambda_{n}\left\|x_{n}-y_{n}\right\|\left[2 \alpha_{n}\left(\gamma\left\|V x_{n}\right\|+\mu\left\|F y_{n}\right\|+\left(2-\alpha_{n}\right)\left\|y_{n}-p\right\|\right)+\lambda_{n}\left\|x_{n}-y_{n}\right\|\right] .
$$

By Step 3, we know that $\lim _{n \rightarrow \infty} M_{n}=0$. Then from (3.22), we have

$$
\begin{aligned}
\left(1-\alpha_{n}\right)\left\|x_{n}-u_{n}\right\|^{2} \leq & \alpha_{n}\left\|\gamma V x_{n}+(I-\mu F) y_{n}-p\right\|^{2} \\
& +\left\|x_{n}-p\right\|^{2}-\left\|x_{n+1}-p\right\|^{2}+M_{n} \\
\leq & \alpha_{n}\left(\gamma\left\|V x_{n}\right\|+\mu\left\|F y_{n}\right\|+\left\|y_{n}-p\right\|\right)^{2} \\
& +\left\|x_{n}-x_{n+1}\right\|\left(\left\|x_{n}-p\right\|+\left\|x_{n+1}-p\right\|\right)+M_{n} .
\end{aligned}
$$

Since $\alpha_{n} \rightarrow 0$ and $\left\|x_{n}-x_{n+1}\right\| \rightarrow 0$, we obtain

$$
\lim _{n \rightarrow \infty}\left\|x_{n}-u_{n}\right\|=0
$$

From Step 3, we also have

$$
\left\|u_{n}-y_{n}\right\| \leq\left\|u_{n}-x_{n}\right\|+\left\|x_{n}-y_{n}\right\| \rightarrow 0, \quad \text { as } n \rightarrow \infty .
$$

Step 5. We show that $\lim _{n \rightarrow \infty}\left\|T u_{n}-u_{n}\right\|=0$, where $T: C \rightarrow H$ is defined by $T x=\lambda x+$ $(1-\lambda) S x$. We know that $T$ is nonexpansive with $F(T)=F(S)$ by Lemma 2.5 . Notice that

$$
\begin{aligned}
\left\|T u_{n}-u_{n}\right\| & \leq\left\|T u_{n}-y_{n}\right\|+\left\|y_{n}-u_{n}\right\| \\
& \leq\left|\lambda-\beta_{n}\right|\left\|u_{n}-S u_{n}\right\|+\left\|y_{n}-u_{n}\right\| .
\end{aligned}
$$

By (3.24) and $\beta_{n} \rightarrow \lambda$, we obtain

$$
\lim _{n \rightarrow \infty}\left\|T u_{n}-u_{n}\right\|=0 .
$$

Step 6. We show that

$$
\limsup _{n \rightarrow \infty}\left\{(\mu F-\gamma V) q, q-x_{n}\right) \leq 0
$$

where $q=P_{F(S) \cap M E P(\Theta, \varphi)}(I-\mu F+\gamma V) q$ is a unique solution of the variational inequality (3.1). To show this inequality, we choose a subsequence $\left\{x_{n_{i}}\right\}$ of $\left\{x_{n}\right\}$ such that

$$
\lim _{i \rightarrow \infty}\left\langle(\mu F-\gamma V) q, q-x_{n_{i}}\right\rangle=\limsup _{n \rightarrow \infty}\left\langle(\mu F-\gamma V) q, q-x_{n}\right\rangle
$$

Since $\left\{u_{n_{i}}\right\}$ is bounded, there exists a subsequence $\left\{u_{n_{i_{j}}}\right\}$ which converges weakly to $w$. Without loss of generality, we can assume that $u_{n_{i}} \rightarrow w$. From Step 4 and Step 5, we obtain 
$x_{n_{i}} \rightarrow w$ and $T u_{n_{i}} \rightarrow w$. By the same argument as in the proof of Theorem 3.1, we have $w \in F(S) \cap \operatorname{MEP}(\Theta, \varphi)$. Since $q=P_{F(S) \cap \operatorname{MEP}(\Theta, \varphi)}(I-\mu F+\gamma V) q$, it follows that

$$
\begin{aligned}
\limsup _{n \rightarrow \infty}\left\langle(\mu F-\gamma V) q, q-x_{n}\right\rangle & =\lim _{i \rightarrow \infty}\left\langle(\mu F-\gamma V) q, q-x_{n_{i}}\right\rangle \\
& =\langle(\mu F-\gamma V) q, q-w\rangle \leq 0 .
\end{aligned}
$$

Step 7. We show that $\lim _{n \rightarrow \infty}\left\|x_{n}-q\right\|=0$, where $q=P_{F(S) \cap M E P(\Theta, \varphi)}(I-\mu F+\gamma V) q$ is a unique solution of the variational inequality (3.1). From (3.14), we know that

$$
x_{n+1}-q=\alpha_{n}\left(\gamma V x_{n}-\mu F q\right)+\lambda_{n}\left(y_{n}-q\right)+\left(\left(1-\lambda_{n}\right) I-\alpha_{n} \mu F\right) y_{n}-\left(\left(1-\lambda_{n}\right) I-\alpha_{n} \mu F\right) q .
$$

Applying Lemma 2.8 and Lemma 2.10, we have

$$
\begin{aligned}
\left\|x_{n+1}-q\right\|^{2} \leq & \left\|\lambda_{n}\left(x_{n}-q\right)+\left(\left(1-\lambda_{n}\right) I-\alpha_{n} \mu F\right) y_{n}-\left(\left(1-\lambda_{n}\right) I-\alpha_{n} \mu F\right) q\right\|^{2} \\
& +2 \alpha_{n}\left\langle\gamma V x_{n}-\mu F q, x_{n+1}-q\right\rangle \\
\leq & \left(\left(1-\lambda_{n}-\alpha_{n} \tau\right)\left\|y_{n}-q\right\|+\lambda_{n}\left\|x_{n}-q\right\|\right)^{2} \\
& +2 \alpha_{n} \gamma\left\langle V x_{n}-V q, x_{n+1}-q\right\rangle+2 \alpha_{n}\left\langle\gamma V q-\mu F q, x_{n+1}-q\right\rangle \\
\leq & \left(1-\tau \alpha_{n}\right)^{2}\left\|x_{n}-q\right\|^{2}+2 \alpha_{n} \gamma l\left\|x_{n}-q\right\|\left\|x_{n+1}-q\right\| \\
& +2 \alpha_{n}\left\langle\gamma V q-\mu F q, x_{n+1}-q\right\rangle \\
\leq & \left(1-\tau \alpha_{n}\right)^{2}\left\|x_{n}-q\right\|^{2}+\alpha_{n} \gamma l\left(\left\|x_{n}-q\right\|^{2}+\left\|x_{n+1}-q\right\|^{2}\right) \\
& +2 \alpha_{n}\left\langle\gamma V q-\mu F q, x_{n+1}-q\right\rangle .
\end{aligned}
$$

This implies that

$$
\begin{aligned}
\left\|x_{n+1}-q\right\|^{2} \leq & \frac{1-2 \tau \alpha_{n}+\tau^{2} \alpha_{n}^{2}+\alpha_{n} \gamma l}{1-\alpha_{n} \gamma l}\left\|x_{n}-q\right\|^{2} \\
& +\frac{2 \alpha_{n}}{1-\alpha_{n} \gamma l}\left\langle\gamma V q-\mu F q, x_{n+1}-q\right\rangle \\
= & \left(1-\frac{2(\tau-\gamma l) \alpha_{n}}{1-\alpha_{n} \gamma l}\right)\left\|x_{n}-q\right\|^{2}+\frac{\tau^{2} \alpha_{n}^{2}}{1-\alpha_{n} \gamma \alpha}\left\|x_{n}-q\right\|^{2} \\
& +\frac{2 \alpha_{n}}{1-\alpha_{n} \gamma l}\left\langle\gamma V q-\mu F q, x_{n+1}-q\right\rangle \\
\leq & \left(1-\frac{2(\tau-\gamma l)}{1-\alpha_{n} \gamma l} \alpha_{n}\right)\left\|x_{n}-q\right\|^{2} \\
& +\frac{2(\tau-\gamma l) \alpha_{n}}{1-\alpha_{n} \gamma l}\left(\frac{\tau^{2} \alpha_{n}}{2(\tau-\gamma l)} M_{2}+\frac{1}{\tau-\gamma l}\left\langle\mu F q-\gamma V q, q-x_{n+1}\right\rangle\right) \\
= & \left(1-\xi_{n}\right)\left\|x_{n}-q\right\|^{2}+\xi_{n} \delta_{n},
\end{aligned}
$$

where $M_{2}=\sup \left\{\left\|x_{n}-q\right\|^{2}: n \geq 1\right\}, \xi_{n}=\frac{2(\tau-\gamma l)}{1-\alpha_{n} \gamma l} \alpha_{n}$ and

$$
\delta_{n}=\frac{\tau^{2} \alpha_{n}}{2(\tau-\gamma l)} M_{2}+\frac{1}{\tau-\gamma l}\left\langle\mu F q-\gamma V q, q-x_{n+1}\right\rangle
$$


From the condition (i) and Step 6, it is easy to see that $\xi_{n} \rightarrow 0, \sum_{n=0}^{\infty} \xi_{n}=\infty$ and $\lim \sup _{n \rightarrow \infty} \delta_{n} \leq 0$. Hence, by Lemma 2.6, we conclude $x_{n} \rightarrow q$ as $n \rightarrow \infty$. This completes the proof.

\section{Remark 3.1}

(1) Theorem 3.1 and Theorem 3.2 extend and develop Theorem 3.1 and Theorem 3.2 of Liu [12], respectively, in the following ways:

(a) The EP (1.2) in Theorem 3.1 and Theorem 3.2 of [12] is extended to the case of the MEP (1.1).

(b) The strongly positive bounded linear operator $A$ in Theorem 3.1 and Theorem 3.2 of [12] is extended to the case of the $\rho$-Lipschitzian and $\eta$-strongly monotone operator $F$. In fact, from the definitions, a strongly positive bounded linear operator $A$ with a constant $\bar{\gamma}>0$ is a $\|A\|$-Lipschitzian and $\bar{\gamma}$-strongly monotone operator.

(c) The contractive mapping $f: H \rightarrow H$ with contractive coefficient $\alpha \in(0,1)$ in Theorem 3.1 and Theorem 3.2 of [12] is extended to the case of a Lipschitzian mapping $V: H \rightarrow H$ with a constant $l \in[0, \infty)$.

(d) The condition $\sum_{n=1}^{\infty}\left|\alpha_{n+1}-\alpha_{n}\right|<\infty$ in Theorem 3.2 of [12] is removed.

(e) The conditions $\sum_{n=1}^{\infty}\left|\beta_{n+1}-\beta_{n}\right|<\infty$ and $\sum_{n=1}^{\infty}\left|r_{n+1}-r_{n}\right|<\infty$ in Theorem 3.2 of [12] are also relaxed by the conditions $\lim _{n \rightarrow \infty}\left|\beta_{n+1}-\beta_{n}\right|=0$ and $\lim _{n \rightarrow \infty}\left|r_{n+1}-r_{n}\right|=0$, respectively.

(2) Even if $C=H, S$ is nonexpansive, $\left\{\beta_{n}\right\}=\{0\}, r_{n}=1, u_{n}=x_{n}, \Theta(x, y)=0$ and $\varphi(x)=0$, $\forall x, y \in C$, Theorem 3.1 and Theorem 3.2 improve Theorem 3.1 and Theorem 3.2 of Tian [18] and Theorem 3.1 and Theorem 3.2 of Ceng et al. [19] from the class of nonexpansive mappings to the class of $k$-strictly pseudocontractive mappings. In particular, Theorem 3.2 develops Theorem 3.2 of Tian [18] and Theorem 3.2 of Ceng et al. [19] by removing the condition either $\sum_{n=1}^{\infty}\left|\alpha_{n+1}-\alpha_{n}\right|<\infty$ or $\lim _{n \rightarrow \infty} \alpha_{n} / \alpha_{n+1}=1$.

(3) Theorem 3.1 also contains Theorem 3.1 of Plubtieng and Pungaeng [13] as a special case with the nonexpansive mapping $S$, and $\left\{\beta_{n}\right\}=\{0\}$, and $\varphi \equiv 0$.

(4) Theorem 3.2 also includes and improves Theorem 3.3 of Plubtieng and Punpaeng [13], Theorem 3.2 of Takahashi and Takahashi [14] as well as Theorem 3.2 of Tian [17], Theorem 2.1 of Jung [8], Theorem 3.1 of Jung [20] Theorem 2.1 of Cho et al. [7] and Theorem 3.4 of Marino and $\mathrm{Xu}[16]$ as some special cases.

Competing interests

The author declares that they have no competing interests.

\section{Acknowledgements}

The author would like to thank the anonymous referees for their valuable comments and suggestions, which improved the presentation of this manuscript. This research was supported by Basic Science Research Program through the National Research Foundation of Korea (NRF) funded by the Ministry of Education, Science and Technology (2012000895).

Received: 25 May 2012 Accepted: 4 October 2012 Published: 17 October 2012

\section{References}

1. Ceng, L-C, Yao, J-C: A hybrid iterative scheme for mixed equilibrium problems and fixed point problems. J. Comput. Appl. Math. 214, 186-201 (2008)

2. Yao, YH, Aslam Noor, M, Zainab, S, Liou, Y-C: Mixed equilibrium problems and optimization problems. J. Math. Anal. Appl. 354, 319-329 (2009) 
3. Blum, E, Oettli, W: From optimization and variational inequalities to equilibrium problems. Math. Stud. 63, 123-145 (1994)

4. Combettes, Pl, Hirstoaga, SA: Equilibrium programming in Hilbert spaces. J. Nonlinear Convex Anal. 6, 117-136 (2005)

5. Flam, SD, Antipin, AS: Equilibrium programming using proximal-like algorithm. Math. Program. 78, $29-41$ (1997)

6. Acedo, GL, Xu, HK: Iterative methods for strictly pseudo-contractions in Hilbert space. Nonlinear Anal. 67, 2258-2271 (2007)

7. Cho, YJ, Kang, SM, Qin, X: Some results on k-strictly pseudo-contractive mappings in Hilbert spaces. Nonlinear Anal. 70, 1956-1964 (2009)

8. Jung, JS: Strong convergence of iterative methods for $k$-strictly pseudo-contractive mappings in Hilbert spaces. Appl. Math. Comput. 215, 3746-3753 (2010)

9. Morales, $\mathrm{CH}$, Jung, JS: Convergence of paths for pseudo-contractive mappings in Banach spaces. Proc. Am. Math. Soc. 128, 3411-3419 (2000)

10. Browder, FE: Fixed point theorems for noncompact mappings. Proc. Natl. Acad. Sci. USA 53, 1272-1276 (1965)

11. Browder, FE, Petryshn, WV: Construction of fixed points of nonlinear mappings Hilbert space. J. Math. Anal. Appl. 20 197-228 (1967)

12. Liu, Y: A general iterative method for equilibrium problems and strict pseudo-contractions in Hilbert spaces. Nonlinear Anal. 71, 4852-4861 (2009)

13. Plubtieng, S, Punpaeng, R: A general iterative method for equilibrium problems and fixed point problems in Hilbert spaces. J. Math. Anal. Appl. 336, 445-468 (2007)

14. Takahashi, A, Takahashi, W: Viscosity approximation methods for equilibrium problems and fixed point problems in Hilbert spaces. J. Math. Anal. Appl. 331, 506-515 (2007)

15. Yamada, I: The hybrid steepest descent for the variational inequality problems over the intersection of fixed points sets of nonexpansive mappings. In: Butnariu, D, Censor, Y, Reich, S (eds.) Inherently Parallel Algorithms in Feasibility and Optimization and Their Applications, pp. 473-504. Elsevier, New York (2001)

16. Marino, G, Xu, HX: A general iterative method for nonexpansive mappings in Hilbert spaces. J. Math. Anal. Appl. 318 43-52 (2006)

17. Tian, M: A general iterative algorithm for nonexpansive mappings in Hilbert spaces. Nonlinear Anal. 73, 689-694 (2010)

18. Tian, M: A general iterative method based on the hybrid steepest descent scheme for nonexpansive mappings in Hilbert spaces. In: 2010 International Conference on Computational Intelligence and Software Engineering, CiSE 2010, art. no. 5677064 (2010)

19. Ceng, L-C, Ansari, QH, Yao, J-C: Some iterative methods for finding fixed points and for solving constrained convex minimization problems. Nonlinear Anal. 74, 5286-5302 (2011)

20. Jung, JS: Some results on a general iterative method for $k$-strictly pseudo-contractive mappings. Fixed Point Theory Appl. 2011, 24 (2011). doi:10.1186/1687-1812-2011-24

21. Peng, J-W, Yao, J-C: A new hybrid-extragradient method for generalized mixed equilibrium problems, fixed point problems and variational inequality problems. Taiwan. J. Math. 12, 1401-1432 (2008)

22. Zhou, H: Convergence theorems of fixed points for $k$-strict pseudo-contractions in Hilbert spaces. Nonlinear Anal. 69 456-462 (2008)

23. Xu, HK: Iterative algorithms for nonlinear operators. J. Lond. Math. Soc. 66, 240-256 (2002)

24. Suzuki, T: Strong convergence of Krasnoselskii and Mann's type sequences for one parameter nonexpansive semigroups without Bochner integral. J. Math. Anal. Appl. 305, 227-239 (2005)

doi:10.1186/1687-1812-2012-184

Cite this article as: Jung: Iterative methods for mixed equilibrium problems and strictly pseudocontractive

mappings. Fixed Point Theory and Applications 2012 2012:184.

\section{Submit your manuscript to a SpringerOpen ${ }^{\circ}$ journal and benefit from:}

- Convenient online submission

Rigorous peer review

- Immediate publication on acceptance

- Open access: articles freely available online

- High visibility within the field

- Retaining the copyright to your article 